\title{
Surface Segregation of Impurities and Hydrogen Transport Kinetics of First Wall Candidate Metals
}

\author{
Kenji YAMAGUCHI and Michio YAMAWAKI \\ Nuclear Engineering Research Laboratory, The University of Tokyo, Tokai-mura, Naka-gun, Ibaraki-ken, 319-11 Japan.
}

(Received on September 27, 1988; accepted in the final form on December 9, 1988)

\begin{abstract}
Surface segregation behaviors of several important metals which are proposed to be used as first wall material of the fusion reactor are briefly reviewed. Examples of such phenomena are presented for nickel, vanadium and stainless steel as were experimentally observed by the present authors. As experimental results showed, the surface phenomena of these metals were characterized initially by the segregation of impurity sulfur. But, in the case of nickel, as sulfur was depleted in the course of experiments due to ion bombardment, the surface was predominated by oxygen which was believed to have been adsorbed from the gas phase. This impurity segregation and adsorption also played an important role on the surface of stainless steel.

The presence of surface impurities due to segregation and/or adsorption is shown here to affect the hydrogen isolope permeation behaviors of these metals. Especially sulfur and oxygen seem to have significant influence on the permeation rate. Strictly speaking, the presence of these impurities are considered to inhibit "hydrogen recombination" process at the surface. And with their strong electronegative properties, some kind of electronic effects must be responsible for lowering recombination coefficient $k_{R}$, where it is demonstrated that accumulation of these impurities decreased $k_{R}$ by orders of magnitude.
\end{abstract}

KEY WORDS: surface segregation; impurities; first wall materials; hydrogen isotopes; permeation; recombination coefficient.

\section{Introduction}

Heating of metals in vacuo induces segregation of certain alloying or non-metal impurity elements on the surface. Such a surface is expected to be quite different from that free from impurities. Therefore, segregation behaviors on metals have been intensively studied in many areas including fusion applications. For instance, alloys of lithium in copper and aluminum have been suggested as possible first wall materials for the purpose of providing low $\mathrm{Z}$ coating. ${ }^{1)}$ It is expected that thermal and/or radiation-induced segregation will produce a lithium-rich overlayer.

Segregation, an increase in the concentration of one or more of the components near lattice discontinuities in alloy systems, ${ }^{2)}$ is in many ways analogous to the phenomenon of adsorption at solid gas interfaces; only the source of the adsorbate is different and the variable is the concentration of the impurity in the bulk instead of gas pressure. As segregation is a thermally-activated process, it becomes significant at elevated temperatures where first walls would be likely to be operated. Furthermore, surface of the first wall would be constantly exposed to irradiation from fucl plasma and also of neutrons, so that an attention must be paid on the effect of irradiation on the segregation and adsorption behaviors.

Permeation of hydrogen isotopes through first wall, on the other hand, will pose a severe problem with respect to recycling, and, when tritium is involved, to contamination of coolant. A transient behavior of hydrogen permeation reveals itself a peculiar phenomenon known as a "permeation spike, $e^{8,4}$ ", in which an initial abrupt increase of the permeation rate of ion-implanted hydrogen is succeeded by its gradual decrease. Several mechanisms have been proposed to explain the spike, and at present it is generally perceived that it is due to the modification of the surface conditions due to ion bombardment. ${ }^{5)}$ Hence, segregation behaviors are likely to have a large impact upon the permeation behaviors.

In this paper, segregation behaviors of impurities at elevated temperatures in such metals as nickel, vanadium and stainless steel which are of interest as candidate materials for the first wall of a fusion reactor are briefly reviewed. Then their impact on the permeation phenomena of hydrogen isotopes is discussed. It has been found by the present authors that the permeation rate of deuterium was intimately correlated with the surface compositions of the metals investigated. ${ }^{6-9)}$ It will be further discussed that the effect of surface impuritics would be to reduce the rate of " hydrogen recombination" process.

\section{Surface Segregation Behaviors of Impurities of Nickel and Vanadium}

\subsection{Nickel}

Nickel foils were obtained from Japan Lamp Industrial Co., Ltd. whose chemical compositions in terms of weight percent was as follows; $\mathrm{Ni}+\mathrm{Co}$; 
$99.45 \%, \mathrm{Cu} ; 0.10 \%, \mathrm{Fe} ; 0.15 \%, \mathrm{Mn} ; 0.20 \%, \mathrm{Si}$; $0.05 \%$, and $\mathrm{S} ; 0.005 \%$. These foils were cut into a circular disc, spot-welded to a sample holder, rinsed with trichloroethylene and then were placed in an ultra-high vacuum vessel. An ultimate background pressure in the vessel was about $1 \times 10^{-7} \mathrm{~Pa}$ at room temperature. When placed in vacuo, surface analysis by means of Auger electron spectroscopy (AES) at room temperature showed that the virgin surface was predominantly covered with carbon and oxygen whose surface concentrations were 58 and 19 at $\%$, respectively, along with a trace of chlorine and sulfur. This surface elemental composition, however, changes drastically due to thermal annealing, ion bombardment, and other surface treatment techniques. In Fig. 1 are shown three Auger electron spectra at $773 \mathrm{~K}$, but taken under different conditions. ${ }^{9)}$ The surface composition begins to alter upon heating the sample over $473 \mathrm{~K}$, as sulfur begins to segregate from the bulk. The surface concentration of sulfur $C_{\mathrm{s}}$ reaches as large as $30 \mathrm{at} \%$ when the temperature is kept at $773 \mathrm{~K}$. An example of such a surface is shown in Fig. 1(a). It is widely known that even a small amount of impurity sulfur in the bulk is sufficient to cause such segregation as shown here. ${ }^{10}$ )

But when deuterium ions with an estimated flux of $1.5 \times 10^{13} \mathrm{D}_{2}^{+} \mathrm{cm}^{-2} \mathrm{~s}^{-1}$ and an incident energy of $3 \mathrm{keV}$

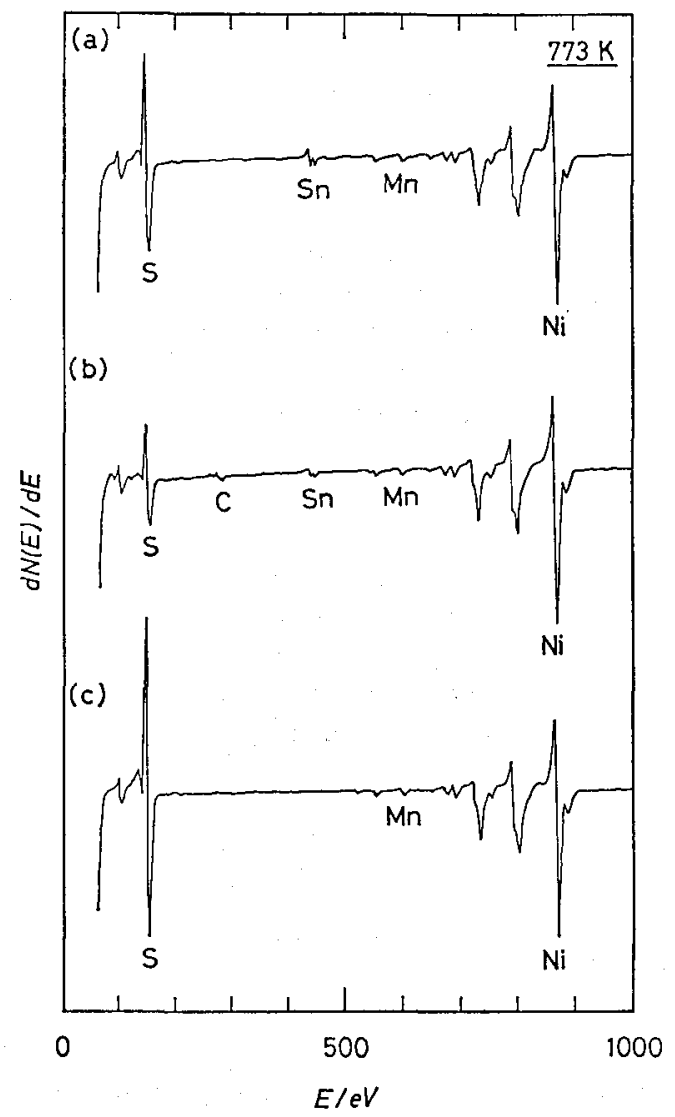

(a) Before $\mathrm{D}_{2}^{+}$ion implantation

(b) After $\mathrm{D}_{2}^{+}$ion implantation for $120 \mathrm{~s}$

(c) After annealing at $923 \mathrm{~K}$ in the hydrogen pressure of $0.5 \mathrm{~Pa}$

Fig. 1. Typical Auger electron spectra of nickel surface at 773 K.91 were implanted into the specimen whose surface composition is represented by Fig. 1(a), $C_{\mathrm{S}}$ decreased to 16 at $\%$ after 2 min of implantation (Fig. 1(b)). This decrease can be attributed to the sputtering effect caused by deuterium ion bombardment. In order to recover the surface composition shown in Fig. 1 (a), at least $10 \mathrm{~h}$ of thermal annealing at $923 \mathrm{~K}$ was necessary to induce sufficient surface segregation of sulfur.

A striking phenomenon was observed when the surface shown in Fig. 1(b) was exposed to pure hydrogen gas with the pressure of about $0.5 \mathrm{~Pa}$ at $923 \mathrm{~K}$. Now, as shown in Fig. 1(c), $C_{\mathrm{S}}$ increased to as large as 40 at $\%$ in less than $30 \mathrm{~min}$. This phenomenon has been also observed in other metals such as platinum and copper, ${ }^{11}$ which, according to those authors, was interpreted as hydrogen " chemisorption-induced" effect.

However, a sulfur-rich surface is not stable permanently under the condition in which it is continuously bombarded by energetic ions. Ion bombardment causes the sputtering of surface atoms, which are then supplied from the bulk. Prolonged ion bombardment in the end leads to considerable depletion of sulfur. Such a phenomenon was experienced by the present authors in the case of nickel. ${ }^{12}$ A result of the surface analysis of that specimen is shown in Figs. 2(a) and 2(b) where the surface concentration of each impurity element $i, C_{i}$, measured before (a) and after (b) $\mathrm{D}_{2}^{+}$ion implantation was plotted against temperature. Note that sulfur was not the predominant surface impurity in this case, but oxygen and silicon were.

\subsection{Vanadium}

A vanadium surface revealed another example of sulfur segregation as is shown in Fig. $3 .{ }^{13)}$ The vana-

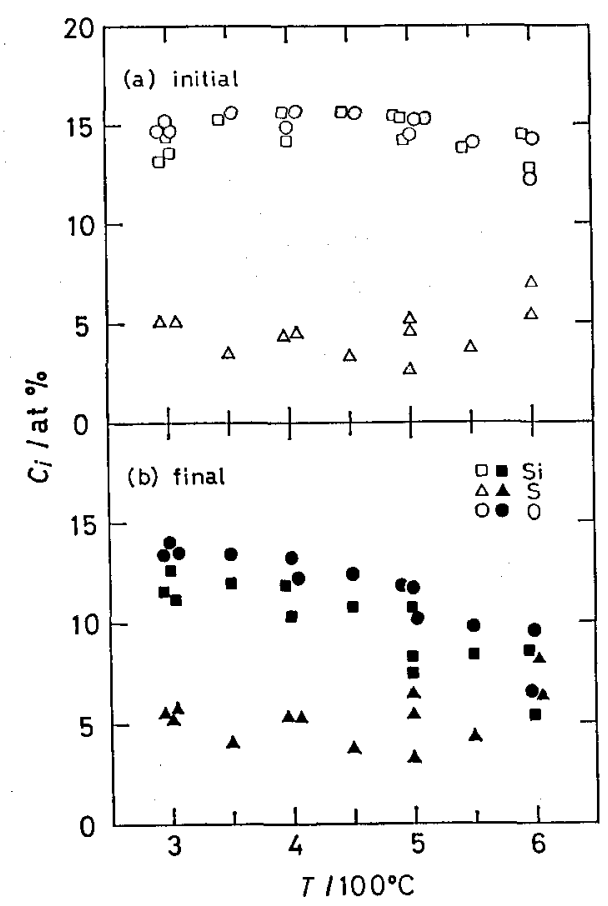

Fig. 2. Composition of the surface impurities $C_{i}$ before and after $\mathrm{D}_{2}^{+}$ion implantation plotted as a function of specimen temperature. ${ }^{12)}$ 


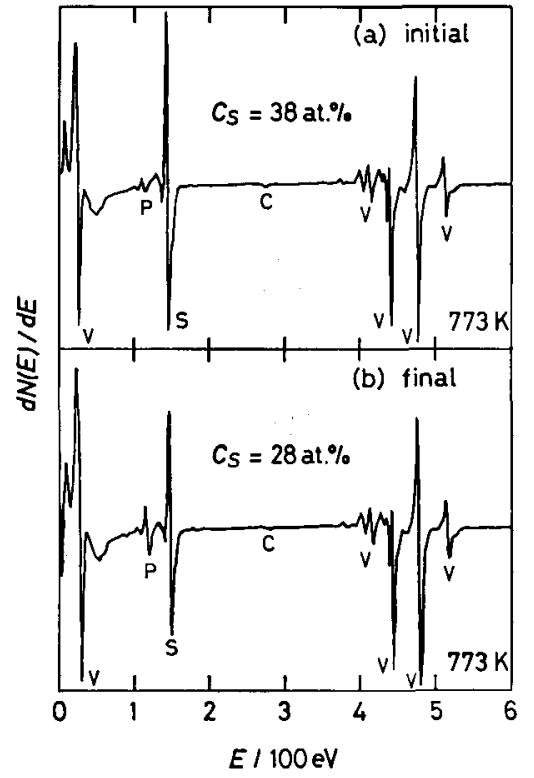

(a) Before $\mathrm{D}_{2}^{+}$ion implantation

(b) After $\mathrm{D}_{2}^{+}$ion implantation

Fig. 3. Auger electron spectra of vanadium surface at $\mathbf{7 7 3}$ $\mathrm{K} .{ }^{13)}$

dium specimen, $99.8 \%$ pure, which was supplied by Goodfellow Metals Ltd., was electrolytically polished in a solution of $80 \%$ methanol and $20 \%$ sulfuric acid. Thickness of the specimen after polishing was $55 \mu \mathrm{m}$. According to a typical AES spectrum of fully annealed surface prior to $\mathrm{D}_{2}^{+}$ion implantation, the surface concentration of sulfur $C_{\mathrm{S}}$ is evaluated to be 38 at $\%$ at $773 \mathrm{~K}$ (Fig. $3(\mathrm{a})$ ). The $C_{\mathrm{s}}$, on the other hand, is reduced to 28 at $\%$ due to about $100 \mathrm{~min}$ of $\mathrm{D}_{2}^{+}$ion implantation (Fig. 3(b)). It should be interesting to note here that this rate of decrease in $C_{\mathrm{s}}$ of vanadium is considerably slow in comparison with that of nickel. This might be due to the difference in the bulk content of sulfur and/or binding energy of sulfur to respective metal atoms on the surface.

The evaluated value of 38 at \% corresponds to fully annealed surface, and is the highest as was obtained in the experiment. This value is higher than the theoretical value of 33 at $\%$ when sulfur is assumed to form $c(2 \times 2)$ structure on $(100)$ surface, ${ }^{14)}$ so that a portion of surface sulfur may be precipitated to form a three-dimensional compound. ${ }^{15}$ ) The same can be said in the case of nickel with $C_{\mathrm{s}}=40 \mathrm{at} \%$.

\section{Impurity Segregation/Adsorption Behaviors of Stainless Steel}

For type 304 stainless steel, the specimen, supplied by Japan Lamp Industrial Co., Ltd. and $50 \mu \mathrm{m}$ in thickness, underwent cycles of ion implantation and subsequent thermal annealing. To be specific, the upstream-side surface of the specimen was initially sputter-cleaned at $973 \mathrm{~K}$ by bombarding $3 \mathrm{keV} \mathrm{Ar}^{+}$ ions, followed by $D_{2}^{+}$ion implantation with estimated flux of $1.9 \times 10^{13} \mathrm{D}_{2}^{+} \mathrm{cm}^{-2} \mathrm{~s}^{-1}$ at $773 \mathrm{~K}$. Then the specimen was thermally annealed at $973 \mathrm{~K}$. In the experiment, this cycle of ion implantation and the

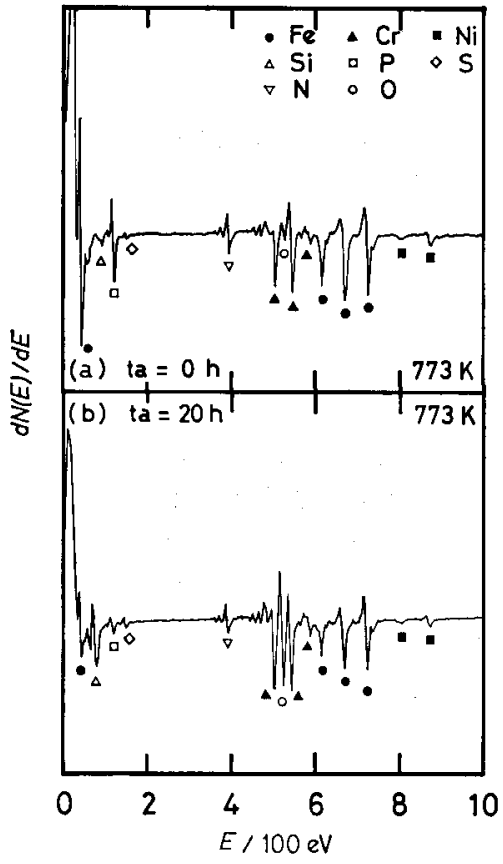

Fig. 4. Auger electron spectra of $304 \mathrm{SS}$ at $773 \mathrm{~K}$ taken from SERIES I.

See text for explanation.

subsequent thermal annealing was repeated until total duration of the annealing at $973 \mathrm{~K}, t_{a}$, exceeded about $20 \mathrm{~h}$. Such a procedure was taken for three series, each differing in the time of initial $\mathrm{Ar}^{+}$sputtering, as will be denoted later by SERIES I to III.

In Fig. 4 are compared two surface compositions belonging to the same series (SERIES I): Fig. 4(a) shows the result of surface analysis at $t_{a}=0 \mathrm{~h}$, and Fig. 4(b) shows that at $t_{a}=20 \mathrm{~h}$. Note that the surface impurity composition significantly altered in the course of annealing. It can be observed that at $t_{a}=$ $0 \mathbf{h}$ the surface is covered with such impurity elements as phosphorus and nitrogen, whereas at $t_{a}=20 \mathrm{~h}$ they are replaced by oxygen and silicon.

The result of the surface analysis is summarized in Fig. 5 where changes in the surface composition of the specimen due to ion implantation and thermal annealing are plotted for two different series, i.e., SERIES I and III. In the figure, open symbols denote the surface concentrations of each impurity element prior to the $\mathrm{D}_{2}^{+}$ion implantation, while solid symbols denote those at the end of implantation. So, the change in surface composition due to $\mathrm{D}_{2}^{+}$ion implantation can be read from that of these two symbols of same $t_{a}$.

According to the figure, the surface analyzed just after $\mathrm{Ar}^{+}$bombardment was covered mostly with phosphorus and nitrogen for both series. But as annealing proceeded, it is interesting to note that while they were replaced by oxygen and silicon rather abruptly in SERIES I, the change in surface composition was very slow in the case of SERIES III.

Owing to sputtering effect, all the impurities present on the surface would be temporarily removed immediately after $\mathrm{Ar}^{+}$sputter-cleaning, so that the surface composition will be determined by the rate of 


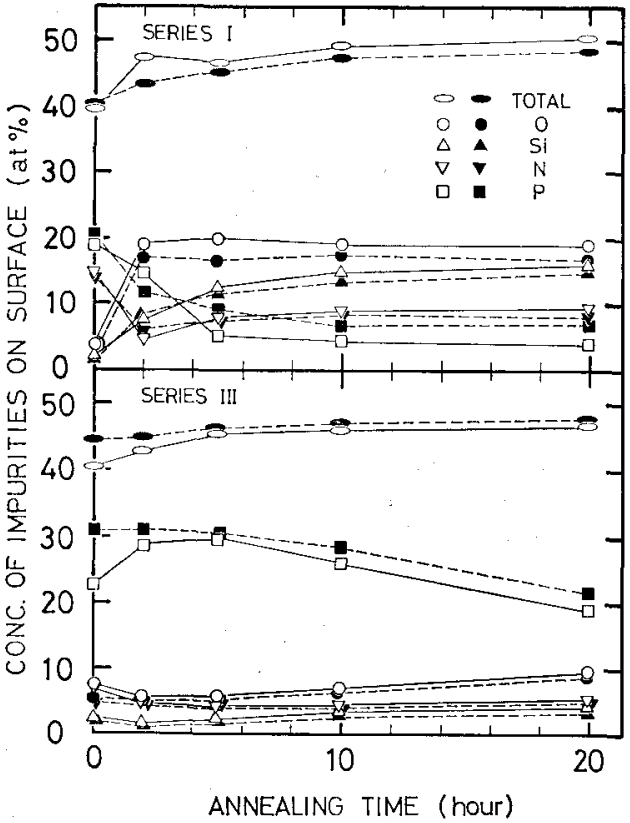

Open symbols: The surface concentrations of respective impurity elements prior to the implantation of deuterium ions

Solid symbols: Those at the end of implantation ${ }^{83}$

Fig. 5. Changes in the surface composition of the specimen at $773 \mathrm{~K}$ due to ion implantation and thermal anneal in the cases of SERIES I (top) and III (bottom).

segregation of each element from the bulk. As Fig. 5 shows, this rate must be larger for phosphorus and nitrogen than oxygen and silicon. This supposition is supported by a thorough study by Yoshihara and $\mathrm{Nii}^{16)}$ in the case of $304 \mathrm{SS}$. However, since the surface covered with oxygen or sulfur, if any, is more favorable from thermochemical standpoint, these impurities will gradually replace the surface sites previously occupied by phosphorus and nitrogen.

As to the origin of oxygen atoms here, some of them may have been dissolved in the bulk, but most of them must have originated from the gas phase. This is clearly shown in Fig. 5. As mentioned above, in SERIES I, an abrupt increase in the surface concentration of oxygen was observed in the range $0 \mathrm{~h}<$ $t_{a}<2 \mathrm{~h}$, but such was not the case in SERIES III. This is the consequence of different annealing procedure employed in those series; while in the latter series the specimen was continuously annealed at 973 $\mathrm{K}$, in the former series it was occasionally cooled down to room temperature at the end of the day even in the middle of thermal annealing. This must have promoted the adsorption, or physisorption, to be exact, of oxygen, and upon heating the specimen, instead of desorbing or dissolving, it formed a stable oxide with chromium and in part with silicon. Evidences for an existence of silicon oxide are as follows:

(1) The shape of silicon in AES spectrum shown in Fig. 4(b) was different from that of pure silicon given in the catalogue, ${ }^{17)}$ and moreover, its location was shifted to the lower energy side.
(2) A correlation of oxygen and silicon was good, as can be seen from Fig. 5 .

(3) A silicon oxide is stable thermochemically.

On the other hand, as to sulfur, which was the predominant impurity species in the cases of nickel and vanadium, it was also observed to segregate on the surface of 304 SS, but only at the initial stage; sulfur was found to quickly be depleted with only a few cycles of ion bombardment and thermal annealing. It seems to be the consequence of adsorption (of oxygen) overwhelming surface segregation (of sulfur), and it is widely known that sulfur on the surface can be removed by exposing it to oxygen..$^{10,18)}$

\section{Significance of Surface Segregation of Im- purities in Fusion Reactors}

\subsection{Correlation between Hydrogen Isotopes Permeation Rate and Surface Compositions}

It has been long pointed out that the permeation behaviors of hydrogen isotopes through metals are in many cases greatly dependent on their surface conditions. Among them, the presence of surface impurities due to segregation and adsorption is believed to be of particular importance. In this sense, experiments on hydrogen isotope permeation have been performed with emphasis on the effect of surface compositions.

Surface compositions, which is characteristic of segregation and adsorption behaviors, were analyzed by means of AES analysis as mentioned before. Permeation rate of hydrogen isotope, be it protium or deuterium, is measured using quadrupole mass spectrometer (QMS) which had been calibrated beforehand. The form of incident hydrogen is $\mathrm{H}_{2}$ or a mixture of $\mathrm{D}_{2}^{+}$and $\mathrm{D}_{2}$, where in the latter case, the $\mathrm{D}_{2}^{+}$ions were accelerated to $3 \mathrm{kV}$ in the ion gun, with the ion flux of about $10^{13}$ ions $\mathrm{cm}^{-2} \mathrm{~s}^{-1}$ in the presence of residual $\mathrm{D}_{2}$ gas of about $10^{-4} \mathrm{~Pa}$ in pressure.

A typical time dependence of permeation rate $\Phi_{P}$ of deuterium observed by QMS is shown in Fig. 6 . When deuterium gas $\mathrm{D}_{2}$ was introduced into the ion gun at $t=0 \mathrm{~min}, \Phi_{P}$ due to that molecular gas was observed. After $\Phi_{P}$ reached a steady state value $\left(\Phi_{b g}\right)_{i}$ at $\sim 100 \mathrm{~min}, \mathrm{D}_{2}^{+}$ions were then implanted. As seen in the figure, a sharp initial rise to $\Phi_{\max }$ is followed by its gradual decrease, which is known as a permeation spike. The implantation was continued until the steady state was established, the rate at which is denoted by $\Phi_{\min }$. After the implantation was terminated, $\left(\Phi_{b q}\right)_{f}$ due to the residual $\mathrm{D}_{2}$ was again observed.

Typically, two AES analyses were performed on the upstream-side surface of the specimen with a permeation experiment in between to give information as to initial and final state of the specimen surface. The permeation rate $\phi_{p}$, expressed as molecules $\mathrm{cm}^{-2}$ $\mathrm{s}^{-1}$, was plotted against surface compositions, namely surface concentration of respective impurity element $i, C_{i}$, and their correlations were investigated.

The result of investigation on 304 SS is summarized 
in Fig. 7 to give an example. As Fig. 4 shows, in the case of 304 SS main constituents of surface impurities were identified as (a) silicon, (b) phosphorus, (c) nitrogen, and (d) oxygen, so that in Fig. 7 the correlations are shown for these elements. In the figure, the permeation rates, $\Phi_{\mathrm{max}}$ and $\Phi_{\mathrm{min}}$, are plotted as a

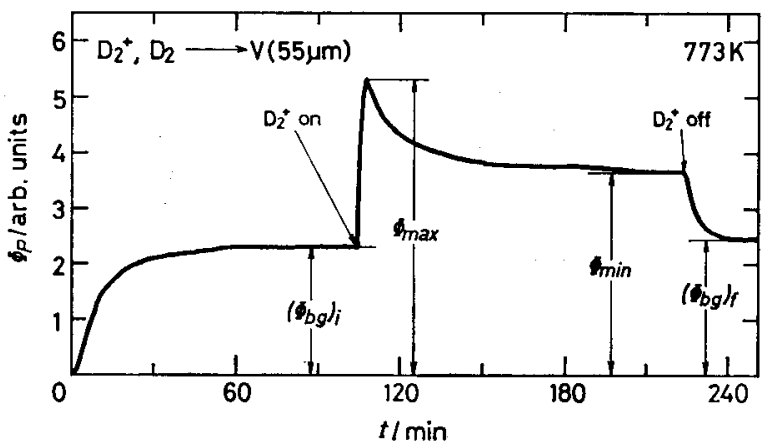

Fig. 6. A typical time $(t)$ dependence of deuterium permeation rate $\Phi_{P}$ for vanadium. ${ }^{13}$ )

Explanation of the symbols are given in the text.
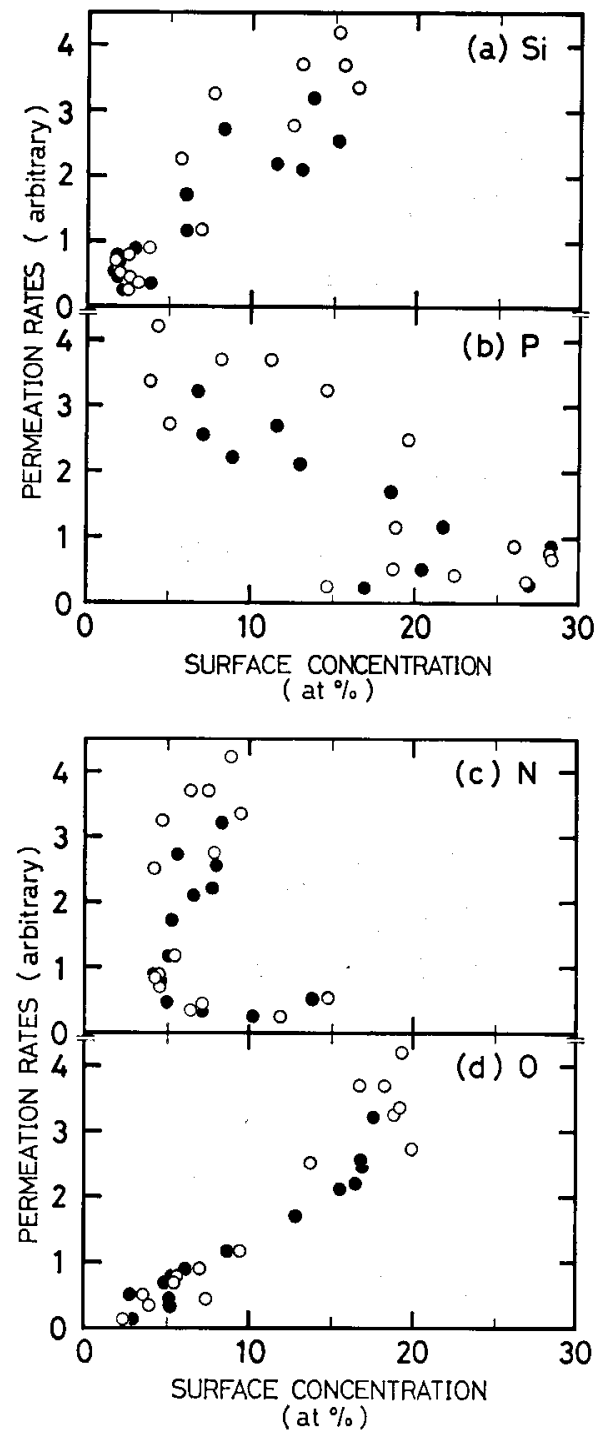

Fig. 7. Relationship of permeation rates to the surface concentration of the impurity element on 304 SS (773 K).

For explanation of the symbols, see text. function of $C_{i}(i=\mathrm{Si}, \mathrm{P}, \mathrm{N}$, or $\mathrm{O})$. It is assumed that $\Phi_{\max }$ can be correlated with the surface state just before being implanted by deuterium ions. This correlation is indicated by an open circle. On the other hand, $\Phi_{\min }$ is assumed to be correlated with the surface state analyzed just after the implantation, and this correlation is denoted by a solid circle. A striking fact is that the correlation was extremely good in the case of oxygen and, though to a lesser degree, to silicon, as shown in Figs. 7 (d) and 7 (a), respectively. The correlation of permeation rate to surface concentration of oxygen was even better than the case when different impurity elements were treated as a whole, the correlation of permeation rate to which is shown in Fig. 8.

As for nickel, it has been found that the correlation was good between the permeation rate and the surface concentration of sulfur $C_{\mathrm{S}}$ as shown in Fig. 9. An open and a solid circle connected with a straight line show that these permeation rates originated from the same experiment. It is interesting to note that the permeation rate is drastically enhanced as $C_{\mathrm{S}}$ exceeds about 30 at $\%$.

\subsection{Hydrogen Recombination Coeffcient and Surface Im- purities}

A model of hydrogen transport in materials based on current understanding is shown in Fig. 10. 19-21)

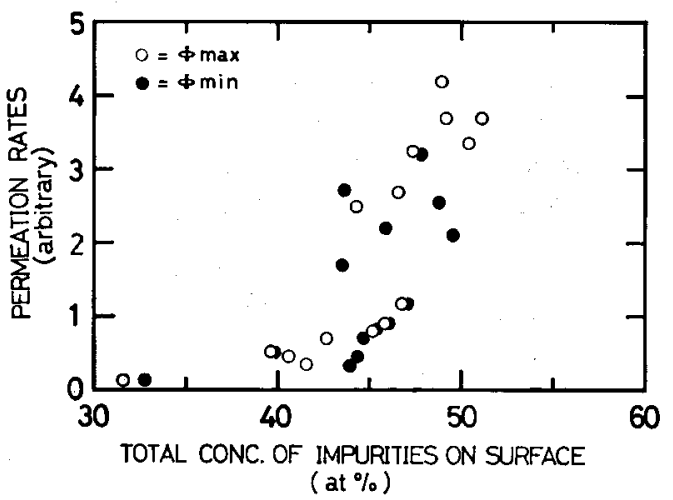

Fig. 8. Relationship of peremation rates to total concentration of impurities on the surface of 304 SS $(773 \mathrm{~K})$.

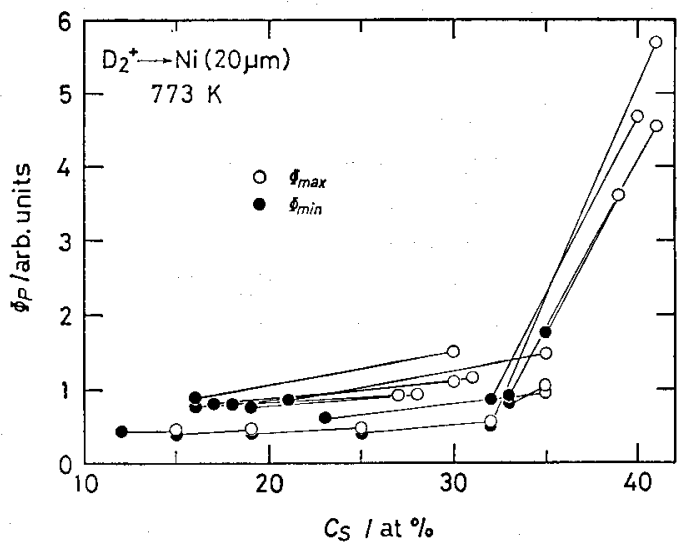

Fig. 9. Relationship between the deuterium permeation rate and surface concentration of sulfur on nickel at 773 K.9) 
Hydrogen is energetically implanted in the form of ion and/or atom, or dissociatively adsorbed from the gas phase. Hydrogen, in the form of atom, forms a concentration profile $C(x)$ and finds its way out via both upstream- and downstream-side surfaces as reemission and permeation, respectively.

In this model are shown two main rate-determining steps of hydrogen transport, namely diffusion in the bulk and hydrogen recombination on the surface. The rate coeflicients for diffusion and recombination process are given in the figure as $D$ and $k_{R}$, respectively, with subscripts 1 and 2 denoting upstreamand downstream-sides, respectively.

A calculation was performed to demonstrate the dependence of deuterium permeation rate $\Phi_{P}$ on recombination coefficients $\left(k_{R}\right)_{1}$ and $\left(k_{R}\right)_{2}$ in the case of nickel at $773 \mathrm{~K}$ whose thickness was $50 \mu \mathrm{m} .{ }^{22)}$ For simplification, $\left(k_{R}\right)_{2}$ was assumed to be constant $\left(10^{-19}\right.$ $\mathrm{cm}^{4} \mathrm{~s}^{-1}$ ) to especially demonstrate the dependence on $\left(k_{R}\right)_{1}$ in the range between $10^{-19}$ and $10^{-15} \mathrm{~cm}^{4} \mathrm{~s}^{-1}$. These values were obtained experimentally as will be mentioned later. And also, a steady state condition was assumed.

The result of calculation is shown in Fig. 11. In the figure is shown for three cases, each differing in the forms of incident deuterium, namely, (i) ion $\mathrm{D}_{2}^{+}$ (ion-driven case), (ii) gas molecule $\mathrm{D}_{2}$ (gas-driven case), and (iii) mixture of both. The incident energy and ion flux of $\mathrm{D}_{2}^{+}$are assumed to be $3 \mathrm{keV}$ and $1.0 \times 10^{13} \mathrm{D}$ atoms cm $\mathrm{cm}^{-2} \mathrm{~s}^{-1}$, respectively, while the gas pressure is assumed to be $1.0 \times 10^{-4} \mathrm{~Pa}$, to represent present authors' typical experimental conditions.

It can be seen, according to the figure, that $\Phi_{P}$

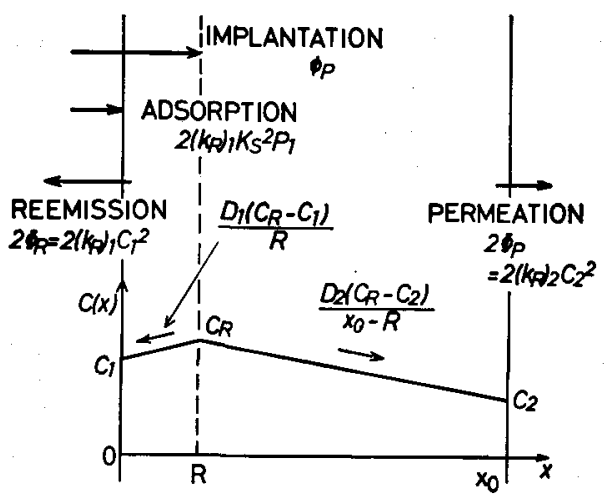

$\phi_{P}:$ Ion or atom flux

$\Phi_{R}, \Phi_{P^{\prime}}:$ Reemission and permeation flux, respectively

$R$ : The projected range of energetic particles, whereas $x_{0}$ is the thickness of the material

$K_{S}, D, k_{R}:$ Solubility, diffusion coefficient, and recombination coefficient, respectively, with subscripts 1 and 2 denoting upstream- and downstream-sides

$C(x):$ A hydrogen atom density profile, where $C_{1}, C_{R}$ and $C_{2}$ are the atomic density at upstream-side surface, range, and downstream-side surface, respectively.

Fig. 10. A model of hydrogen isotope transport in material. tends to decrease in ion-driven case, but to increase in gas-driven case with increasing $\left(k_{R}\right)_{1}$ each. This is the consequence of the fact that low values of $\left(k_{R}\right)_{1}$ imply that number of molecules entering the material is decreased due to the slow process of adsorption. Whereas in ion-driven case they imply that the reemission rate is reduced, thereby increasing the permeation rate, according to the steady state model shown in Fig. 10, as surface barrier is utterly ineffective against entering of energetic particles.

A main reason for varying recombination coefficient $k_{R}$ is that it has been considered to be greatly affected owing to the presence of surface impurities. The reported values of $k_{R}$ by many authors differ by orders of magnitude, and this has been attributed to the presence of surface impurities. ${ }^{23)}$

The dependence of $k_{R}$ on surface composition has been thoroughly investigated by the present authors. ${ }^{9,12,13)}$ An example of such is shown for nickel in Fig. 12, where $\left(k_{R}\right)_{1}$ is plotted as a function of surface concentration of sulfur $C_{\mathrm{s}} . \quad\left(k_{R}\right)_{1}$ is evaluated

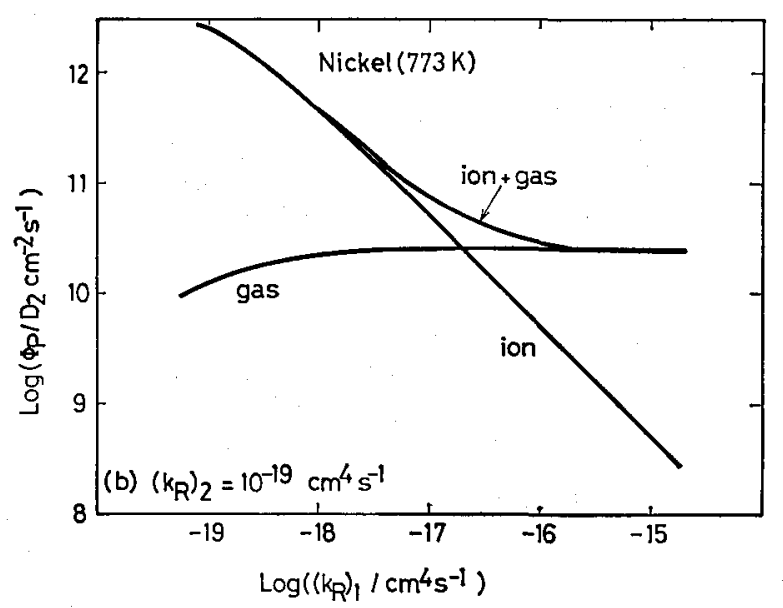

Fig. 11. Permeation rate of deuterium through nickel $(50$ $\mu \mathrm{m}$ in thickness) at $773 \mathrm{~K}$, calculated as a function of $\left(k_{R}\right)_{1}$, assuming $\left.\left(k_{R}\right)_{2}=10^{-19} \mathrm{~cm}^{4} \mathrm{~s}^{-1} .22\right)$

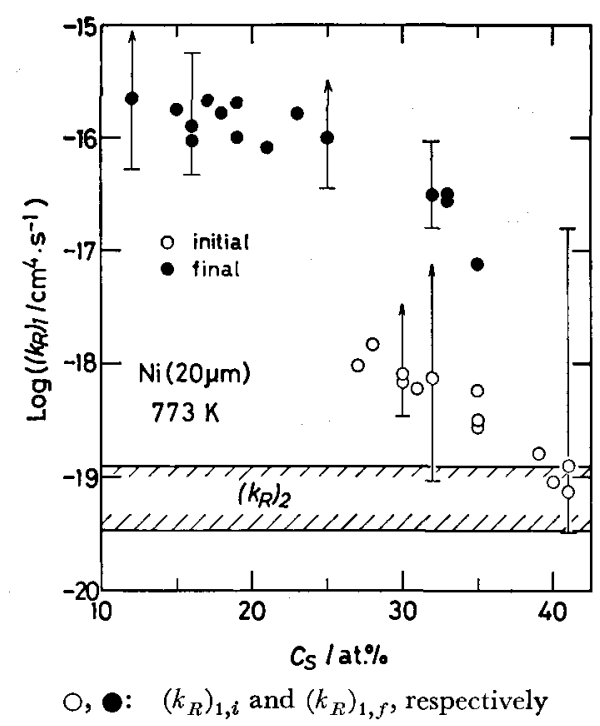

Fig. 12. Recombination coefficient of nickel experimentally determined as a function of surface concentration of sulfur $C_{\mathrm{S}}$ at $773 \mathrm{~K}$. 
by solving the following equations (see also Fig. 10 for explanation of the symbols);

$$
\begin{aligned}
& \left(k_{R}\right)_{2}= \\
& \frac{\Phi_{\min }}{\left[\left(\frac{\left(\phi_{P} / 2-\Phi_{\min }\right)}{\left(k_{R}\right)_{1, f}}+K_{S}^{2} P_{1}\right)^{1 / 2}-\frac{\left(2 x_{0} \Phi_{\min }-R \phi_{P} / 2\right)}{D}\right]^{2}}, \\
& \left(k_{R}\right)_{2}=\frac{\left(\Phi_{b q}\right)_{f}}{\left[\left(K_{S}^{2} P_{1}-\frac{\left(\Phi_{b q}\right)_{f}}{\left(k_{R}\right)_{1, f}}\right)^{1 / 2}-\frac{2 x_{0}\left(\Phi_{b q}\right)_{f}}{D}\right]^{2}}, \\
& \left(k_{R}\right)_{1, i}=\frac{\left(\Phi_{b q}\right)_{f}}{K_{S}^{2} P_{1}-\left[\left(\frac{\left(\Phi_{b_{q}}\right)_{f}}{\left(k_{R}\right)_{2}}\right)^{1 / 2}+\frac{2 x_{0}\left(\Phi_{b g}\right)_{f}}{D}\right]^{2}} \text {. }
\end{aligned}
$$

These equations are solved for $\left(k_{R}\right)_{1, i},\left(k_{R}\right)_{1, f}$ and $\left(k_{R}\right)_{2}$ where $\left(k_{R}\right)_{1, i}$ and $\left(k_{R}\right)_{1, f}$ are initial and final values of $\left(k_{R}\right)_{1}$, and in Fig. 12 they are denoted by an open and a solid circle, respectively. As the figure shows, there occurred an appreciable error in the evaluation of $\left(k_{R}\right)_{1}$ 's, whereas that of $\left(k_{R}\right)_{2}$ was smaller as shown by a hatched area.

Still, it can be seen that $\left(k_{R}\right)_{1}$ decreased by about four orders of magnitude with increasing $C_{\mathrm{s}}$. Together with Fig. 11, it can be concluded that the presence of surface impurities on the upstream-side enhances the iron-driven permeation of hydrogen to the other side. This is exactly what was explained in Fig. 9.

$k_{R}$ was also found to decrease with increasing $C_{\mathrm{S}}$ in the case of vanadium, ${ }^{13)}$ and in the case of 304 SS it was found to decrease with increasing the surface concentration of oxygen. ${ }^{24)}$

\subsection{The Role of Surface Impurities}

Now the role of the surface impurities on hydrogen transport, recombination process, to be specific, has to be examined. As the Figs. 7 to 9 show, the fact that the accumulation of surface impurities on upstream-side as a whole increased iron-driven permeation rate implies that they are effective in suppressing the reemission flux (see Fig. 10) by blocking the surface sites for hydrogen, thereby increasing permeation flux to the downstream-side. However, as Fig. 7(b) or 7 (c) shows for some impurities, the permeation rate is negatively or has virtually no appreciable correlation with them, in the presence of others. Hence, it can be deduced that it is the chemical state of the surface characterized by certain impurity elements that determines the permeation behaviors. Both sulfur and oxygen have very strong electronegative properties. They are known to strip electrons from metal atoms to cause an increase in work function, ${ }^{25-27)}$ and in general act as poisons for adsorption process, whereas the electropositive elements, such as sodium, potassium and cesium, act as promoters. ${ }^{28)}$ Then this surface charge redistribution due to the accumulation of impurities should be responsible for inhibiting hydrogen dissociative adsorption and recombination process.

As to the role of above impurities on hydrogen adsorption and recombination on an atomic scale, some kind of electronic effects has to be considered, but there seem to be two contradictory explanations. One is that the role of the sulfur on the surface would be to prevent the dissociation of hydrogen by blocking surface sites of hydrogen. ${ }^{29-31}$ ) This supposition is supported by the experimental result that there was no adsorption of $\mathrm{H}_{2}$ on $\mathrm{Ag}(100)$ at $160 \mathrm{~K}$ with predosed oxygen as determined by means of thermal desorption measurements between 160 and $1000 \mathrm{~K} .{ }^{32)}$ As the adsorption studies of hydrogen on clean palladium show, the dissociative adsorption gives rise to an increase of the work function. ${ }^{33,34)}$ This indicates that a net electron transfer from the metal to hydrogen atom, so that a negatively charged hydrogen layer will be formed.

Another possibility is that hydrogen is strongly bonded to oxygen atom to form hydroxide species on the surface. ${ }^{35-38)}$ In this case, hydrogen must be positively charged. This is, however, incompatible with the experimental results mentioned just above, and still not justified for sulfur, but, when taking into account the "chemisorption-induced" segregation as was mentioned in Sec. 2.1, one can not ignore the possibility of there existing strong interaction between hydrogen and sulfur.

\section{Summary}

As the present review has shown, surface segregation behaviors of nickel and vanadium were characterized by accumulation of sulfur up to as high as 40 at $\%$ as determined by Auger electron spectroscopy. This sulfur segregated surface is considered to be chemically stable and it continues to be so, so long as the bulk content of sulfur holds; it was shown that repeated cycles of ion bombardment and thermal annealing reduced surface segregation of sulfur. As for stainless steel (304 SS), on the other hand, the adsorption of oxygen in the gas phase was so strong as to replace all other segregated impurities with the exception of silicon which showed great affinity toward oxygen, probably forming an oxide.

In fusion reactor, permeation of hydrogen isotopes may pose a severe problem with respect to fuel particle balance. It was shown here in the cases of 304 SS and nickel that permeation rate of deuterium was intimately correlated with surface impurity compositions, which were characteristics of surface segregation and adsorption: for 304 SS it was oxygen that governed the permeation rate, whereas for nickel it was sulfur.

The significance of surface impurities is that they inhibit hydrogen recombination process which is one of the principal rate-determining steps of the hydrogen transport in materials. In the present review, hydrogen recombination coefficient $k_{R}$ was derived using experimentally obtained permeation data, and was evaluated as a function of surface impurity compositions. The results were that $k_{R}$ decreased with increasing the surface concentration of oxygen in the case of $304 \mathrm{SS}$, and of sulfur in the cases of nickel and vanadium.

In discussing the role of these impurities on an 
atomic scale, it is certain that some kind of electronic effects are involved but the study in this reagrd is still inadequate at present, so it is too early to draw any further conclusion than described in the manuscript.

Before concluding, reference should be made to the irradiation effect when applying above metals in future fusion devices. Considering edge plasma in the vicinity of first wall, hydrogen energy and flux would be $<100 \mathrm{eV}$ and $>10^{16} \mathrm{H} \mathrm{cm}^{-2} \mathrm{~s}^{-1}$, respectively. They are by far away from the experimental conditions of the present authors, so that naturally their future strategy will be to perform studies on "low energy" and "high flux" conditions. At present therc is virtually no knowledge as to the effect of radiation damage on hydrogen recombination process, and also so-called "radiation-enhanced" effect on segregation behavior is still not adequately understood. The dynamical change of surface conditions would surely be reflected upon $k_{R}$, which in turn would affect hydrogen isotopes permeation behaviors.

\section{REFERENCES}

1) D. M. Gruen, A. R. Krauss, S. Susman, M. Venugopalan and M. Ron: J. Vac. Sci. Technol. A, 1 (1983), 924.

2) J. C. Shelton, H. R. Patil and J. M. Blakely: Surf. Sci., 43 (1974), 493.

3) H. K. Perkins and T. Noda: J. Nucl. Mater., 71 (1978), 349.

4) T. Tanabe, N. Saito, Y. Etoh and S. Imoto: J. Nucl. Mater., 103 \& 104 (1981), 483.

5) R. A. Kerst and W. A. Swansiger: J. Nucl. Mater., 122 \& 123 (1984), 1499.

6) M. Yamawaki, T. Namba, T. Kiyoshi, T. Yoneoka and M. Kanno: J. Nucl. Mater., 133 \& 134 (1985), 292.

7) M. Yamawaki, T. Kiyoshi, T. Namba and M. Kanno: Z. Phys. Chem. Neue. Folge, 147 (1986), 115.

8) M. Yamawaki, K. Yamaguchi, T. Kiyoshi and T. Namba: J. Nucl. Mater., 145-147 (1987), 309.

9) M. Yamawaki, T. Namba, K. Yamaguchi and T. Kiyoshi: Nucl. Instr. Meth., B23 (1987), 498.

10) L. Harris: J. Appl. Phys., 39 (1968), 1428.

11) I. Szymerska and M. Lipski: J. Catal., 47 (1977), 144.

12) K. Yamaguchi, T. Namba and M. Yamawaki: J. Nucl. Sci. Technol., 24 (1987), 915.
13) K. Yamaguchi, M. Yamawaki, T. Kiyoshi and T. Namba: Proc. of the 8th Int. Symp. on Plasma Chemistry, Int. Union Pure Appl. Chem., (1987), 1626.

14) L. Fiermans and J. Vennik: Surf. Sci., 24 (1971), 541.

15) K. Nii and K. Yoshihara: J. Jpn. Inst. Metals, 44 (1980), 100.

16) K. Yoshihara and K. Nii: J. Jpn. Inst. Metals, 46 (1982), 494.

17) Auger Electron Spectra Catalogue, Anelva Corp., (1979).

18) P. H. Holloway and J. B. Hudson: Surf. Sci., 33 (1972), 56.

19) B. L. Doyle: J. Nucl. Mater., 111 \& 112 (1982), 628.

20) B. L. Doyle and D. K. Brice: J. Nucl. Mater., 145-147 (1987), 288.

21) F. Waelbroeck: KFA report, Jul-1966 (1984), and references therein.

22) K. Yamaguchi, T. Namba, S. Tanaka and M. Yamawaki: to be published in Fusion Engineering and Design, (1989).

23) R. A. Causey and M. I. Baskes: Proc. of the Int. Symp. on Fusion Reactor Blanket and Fuel Cycle Technology, Univ. of Tokyo, Tokyo, (1986), 177, and references therein.

24) M. Yamawaki, K. Yamaguchi, S. Tanaka, T. Namba, T. Kiyoshi and Y. Takahashi: to be published in J. Nucl. Mater.

25) M. Blaszczyszyn, R. Blaszczyszyn, R. Meclewski, A. J. Melmed and T. F. Madey: Surf. Sci., 131 (1983), 433.

26) G. Panzner and B. Egert: Surf. Sci., 144 (1984), 651.

27) E. L. Hardegrec, P. Ho and J. M. White: Surf. Sci., 165 (1986), 488.

28) J. K. Nørskov, S. Holloway and N. D. Lang: Surf. Sci., 137 (1984), 65.

29) E. I. Ko and R. J. Madix: Surf. Sci., 100 (1980), L449.

30) E. I. Ko and R. J. Madix: Surf. Sci., 109 (1981), 221.

31) G. M. Pradier, Y. Berthier and J. Oudar: Surf. Sci., 130 (1983), 229.

32) M. Bowker, M. A. Barteau and R. J. Madix: Surf. Sci., 92 (1980), 528.

33) H. Conrad, G. Ertl and E. E. Latta: Surf. Sci., 41 (1974), 435 .

34) R. J. Behm, K. Christmann and G. Ertl: Surf. Sci., 99 (1980), 320.

35) A. Benninghoven, K. H. Mueller, C. Plog, M. Schemmer and P. Steffens: Surf. Sci., 63 (1977), 403.

36) A. Benninghoven, K. H. Mueller and M. Schemmer: Surf. Sci., 78 (1978), 565.

37) A. Benninghoven, P. Beckmann, D. Greifendorf, K. H. Mueller and M. Schemmer: Surf. Sci., 107 (1981), 148.

38) J. S. Villarrubia and W. Ho: Surf. Sci., 144 (1984), 370. 